The Astrophysical Journal, ACCEPTED

Preprint typeset using LATEX style emulateapj v. 8/13/10

\title{
NON-EQUILIBRIUM ELECTRONS IN THE OUTSKIRTS OF GALAXY CLUSTERS
}

\author{
Camille Avestruz ${ }^{1,2}$, Daisuke Nagai ${ }^{1,2,3}$, ERWIn T. LAU ${ }^{1,2}$, AND KAYlea Nelson ${ }^{2,3}$ \\ ${ }^{1}$ Department of Physics, Yale University, New Haven, CT 06520, U.S.A.; camille.avestruz@yale.edu \\ ${ }^{2}$ Yale Center for Astronomy \& Astrophysics, Yale University, New Haven, CT 06520, U.S.A. \\ ${ }^{3}$ Department of Astronomy, Yale University, New Haven, CT 06520, U.S.A.
}

The Astrophysical Journal, accepted

\begin{abstract}
The analysis of X-ray and Sunyaev-Zel'dovich measurements of the intracluster medium (ICM) assumes that electrons are in thermal equilibrium with ions in the plasma. However, in the outskirts of galaxy clusters, the electron-ion equilibration timescale can become comparable to the Hubble time, leading to systematic biases in cluster mass estimates and mass-observable scaling relations. To quantify an upper limit of the impact of nonequilibrium electrons, we use a mass-limited sample of simulated galaxy clusters taken from a cosmological simulation with a two-temperature model that assumes the Spitzer equilibration time for the electrons and ions. We show that the temperature bias is more pronounced in more massive and rapidly accreting clusters. For the most extreme case, we find that the bias is of order $10 \%$ at half of the cluster virial radius and increases to $40 \%$ at the edge of the cluster. Gas in filaments is less susceptible to the non-equilibrium effect, leading to azimuthal variations in the temperature bias at large cluster-centric radii. Using mock Chandra observations of simulated clusters, we show that the bias manifests in ultra-deep X-ray observations of cluster outskirts and quantify the resulting biases in hydrostatic mass and cluster temperature derived from these observations. We provide a mass-dependent fitting function for the temperature bias profile, which can be useful for modeling the effect of electron-ion equilibration in galaxy clusters.
\end{abstract}

Subject headings: cosmology: theory — galaxies:clusters: general — galaxies:clusters:intracluster medium — methods : numerical $-\mathrm{X}$-rays:galaxies:clusters

\section{INTRODUCTION}

Galaxy clusters constrain cosmology, but the power of cluster-based cosmology is limited by our understanding of astrophysical processes that govern cluster formation. The evolution of cluster abundance is particularly sensitive to cosmological parameters (e.g. Allen et al.2011, for review). Recent advances in X-ray and microwave observations have enabled measurements of properties of the intracluster medium (ICM) out to the virial radius and produced cosmological constraints that are complementary to other probes (Vikhlinin et al. 2009; Mantz et al. 2010; Planck Collaboration et al. 2014).

However, recent X-ray and microwave measurements at the largest radii have exhibited unexpected features, which raise a question whether we understand the global ICM properties well enough to use galaxy clusters for a robust cosmological probe. First, several entropy profiles measured with the Suzaku X-ray observatory showed flattening outside of $R_{500 d}{ }^{1}$ (e.g., Bautz et al. 2009; Reiprich et al. 2009, Hoshino et al. 2010, Kawaharada et al. 2010; Walker et al. 2013; Urban et al. 2014), deviating from theoretical predictions from hydrodynamical simulations (Tozzi \& Norman 2001; Voit et al. 2003). Second, Suzaku X-ray measurements of the Perseus cluster revealed an enclosed gas mass fraction that exceeds the cosmic baryon fraction (Simionescu et al.|2011). Last, Xray follow-up observations of high redshift clusters selected by Sunyaev-Zel'dovich (SZ) surveys found ICM temperatures lower than the theoretical self-similar predictions in the outer regions of clusters (McDonald et al. 2014). Several possible astrophysical phenomena have been proposed to explain

\footnotetext{
${ }^{1} R_{500 c}$ is the cluster radius enclosing an average density 500 times the critical density of the universe.
}

the discrepancy between theoretical predictions and observations, including gas clumping (Nagai \& Lau 2011, Zhuravleva et al. 2013; Vazza et al. 2013, Roncarelli et al.||2013) and non-thermal pressure support (e.g. Nelson et al. 2014a Shi \& Komatsu 2014; Shi et al. 2014). Another potentially important astrophysical process that can lead to biased gas measurements in the outskirts of clusters is the temperature non-equilibrium between electrons and the heavier ions in the intracluster plasma (Fox \& Loeb 1997; Ettori \& Fabian|1998 Wong \& Sarazin 2009, Rudd \& Nagai 2009).

Collisionless shocks convert the bulk kinetic energy of each particle species into thermal energy. Heavier ions possess most of the bulk kinetic energy and therefore retain most of the thermal energy in the post-shock gas. The shock heats the electrons to a much lower temperature, proportional to the temperature of the heavier ions by a factor of the ratio of their masses $m_{e} / m_{i}$. Electrons are subsequently heated by the ions via Coulomb collisions and other interactions. If Coulomb collisions are the main physical mechanism that equilibrates electron and ion temperatures in cluster outskirts, equilibration timescales can be very long, leaving electrons at colder temperatures than the mean temperature of the gas.

Since X-ray and SZ observations are sensitive to the electron component of the intracluster plasma, non-equilibrium electrons could introduce a bias in derived physical properties of the ICM in cluster outskirts. Derived ICM properties assume that the measured electron temperature corresponds to the total thermal energy of the plasma. However, the mean gas temperature, which is an average over all particle species, may be higher than the electron temperature. To understand the origins of the tension between theoretical predictions and cluster outskirt observations, it is important to distinguish and quantify the relative importance of non-equilibrium electrons 
from other effects.

By analyzing three simulated clusters extracted from cosmological simulations with the two-temperature effect, Rudd \& Nagai (2009) showed that the bias in electron temperature is dependent on the mass and dynamical state of a galaxy cluster. More massive clusters have higher temperatures that lead to longer equilibration time scales, while more disturbed clusters have undergone recent mergers that generate more nonequilibrium electrons.

In this work, we quantify the temperature bias due to nonequilibrium electrons using a statistical sample of galaxy clusters from a high-resolution cosmological simulation of galaxy clusters. We first establish and quantify the statistical relationship between the temperature bias and both the cluster mass and mass accretion rate (MAR). By analyzing mock Chandra observations of simulated clusters, we assess the nonequilibrium effects on the ICM temperatures measured from observations. We discuss implications for future X-ray and SZ measurements of cluster outskirts.

Our paper is organized as follows. In Section 2 we briefly describe the simulations we used and the mock Chandra analysis pipeline. We present our results in Section 3 , and give our summary and discussion in Section 4

\section{METHODOLOGY}

\subsection{Cosmological Simulation with a Two Temperature Model}

We use a mass-limited sample of 65 galaxy clusters from a high-resolution cosmological simulation Omega500, the details of which can be found in Nelson et al. (2014b). The simulation box is $500 \mathrm{~h}^{-1} \mathrm{Mpc}$ on each side, and has a peak spatial resolution of $3.8 h^{-1} \mathrm{kpc}$. We neglect radiative cooling and star formation, which should have minimal effects on cluster outskirts.

Using the Adaptive Refinement Tree code (Kravtsov 1999 Kravtsov et al. 2002; Rudd et al. 2008), we performed the simulation with a modification to model electrons and heavier ions separately as described in Rudd \& Nagai (2009). Electrons and ions are assumed to be in local thermodynamic equilibrium with separate respective temperatures $T_{e}$ and $T_{i}$, and the relaxation process between the two components is explicitly calculated. The electron temperature time evolution is modeled as,

$$
\frac{d T_{e}}{d t}=\frac{T_{e}-T_{i}}{t_{e i}}-(\gamma-1) T_{e} \nabla \cdot \mathbf{v},
$$

where the second term accounts for heating and cooling from adiabatic accretion, $\gamma=5 / 3$ is the adiabatic index, $\mathbf{v}$ is the gas velocity, and $t_{e i}$ is the equilibration timescale for a fully ionized medium (Spitzer 1962) comprised of electrons, protons, and He II,

$$
t_{e i}=6.3 \times 10^{8} \mathrm{yr}\left(\frac{T_{e}}{10^{7} \mathrm{~K}}\right)\left(\frac{n_{i}}{10^{-5} \mathrm{~cm}^{-3}}\right)^{-1}\left(\frac{\ln \Lambda}{40}\right)^{-1},
$$

where $n_{i}$ is the ion number density, and $\ln \Lambda$ is the Coulomb logarithm,

$$
\ln \Lambda=37.8+\ln \left(\frac{T_{e}}{10^{7} \mathrm{~K}}\right)-\frac{1}{2} \ln \left(\frac{n_{i}}{10^{-5} \mathrm{~cm}^{-3}}\right) .
$$

In this two-temperature model, the hydrodynamic equations are computed using the total thermal energy of the gas and the electron temperature is a passively evolving scalar field whose evolution is described by Equation 1 . We do not keep track of potential temperature differences between heavier ions, such as hydrogen and helium ions, since the equilibration timescales between heavier ions are very short compared to that of the electrons; i.e., the equilibration timescale between He II and protons is a factor of $\sqrt{m_{e} / m_{\mathrm{HeII}}}$ of the equilibration timescale between electrons and protons.

Accretion shocks thermalize the electrons and ions accreting from the cosmic web. The heavier ions acquire most of the thermal energy, and heat the electrons through Colulomb collisions in the post-shock regions. However, in the diffuse cluster outskirts with gas temperatures around $T>10^{7} \mathrm{~K}$ and densities between 10-100 times the cosmic mean density, $t_{e i}$ becomes comparable to the Hubble time, causing the electron temperatures to be lower than the ion temperatures.

The Spitzer timescale adopted in our simulations is an upper limit of the true equilibration time, as there are other physical mechanisms such as plasma instabilities (e.g., Bykov et al. 2008) that can couple the temperatures of the different ion species. However, the effects of plasma instabilities are expected to be small in the high Mach number accretion shocks, which are responsible for generating the nonequilibrium electrons in cluster outskirts. Plasma instabilities may provide additional non-adiabatic heating that shortens the equilibration timescale in gas with lower Mach numbers. Therefore, the results in this paper should be taken as a limit on the maximal effects of non-equilibrium electrons.

\subsection{Mock Chandra Analysis}

We create realistic mock X-ray photon maps from X-ray flux maps by convolving with Chandra response files. We generate two sets of X-ray flux maps: (1) the first uses a projected X-ray emissivity that has been generated using the mean gas temperature in the simulation $T_{\text {gas }}=\left(n_{i} T_{i}+\right.$ $\left.n_{e} T_{e}\right) /\left(n_{i}+n_{e}\right)$, where $n_{e}$ is the electron density; and (2) the second uses the electron temperature, $T_{e}$.

Below we summarize the main elements of the mock Chan$d r a$ analysis pipeline here. Further details of the pipeline can be found in Nagai et al. (2007) and Avestruz et al. (2014).

The X-ray emissivity for a given $k$-th hydrodynamical cell with volume $\Delta V_{k}$ in the simulation is given by,

$$
j_{E, k}=n_{e, k} n_{i, k} \Lambda_{E}\left(T_{k}, Z_{k}, z\right) \Delta V_{k},
$$

where $n_{e}$ and $n_{i}$ are the respective number densities of electrons and ions, $T_{k}$ is either the mean gas temperature $\left(T_{\mathrm{gas}}\right)$ or the electron temperature of that element $\left(T_{e}\right), Z_{k}=0.3 Z_{\odot}$ is the assumed metallicity, and $z$ is the redshift. We assume a constant metallicity that is representative of a plausible average metallicity for a galaxy cluster, since our simulation does not follow cooling and star formation. We compute the Xray emissivity, $\Lambda_{E}\left(T_{k}, Z_{k}, z\right)$, using the MEKAL plasma code (Mewe et al. 1985, Kaastra \& Jansen 1993, Liedahl et al. 1995). We multiply the plasma spectrum by the Galactic absorption corresponding to a hydrogen column density of $N_{\mathrm{H}}=2 \times 10^{20} \mathrm{~cm}^{-2}$.

We convolve the emission spectrum with the response of the Chandra ACIS-I CCDs and draw photons from each position and spectral channel according to Poisson distribution. The resulting photon maps have an exposure time of $2.4 \mathrm{Msec}$, similar to the deep Chandra observations of Abell 133 (Vikhlinin et al., in prep.), and comparable in photon counts to stacked galaxy cluster analyses. From images generated in the $0.7-2 \mathrm{keV}$ band, we identify and mask out clumps using the wavelet decomposition algorithm described 

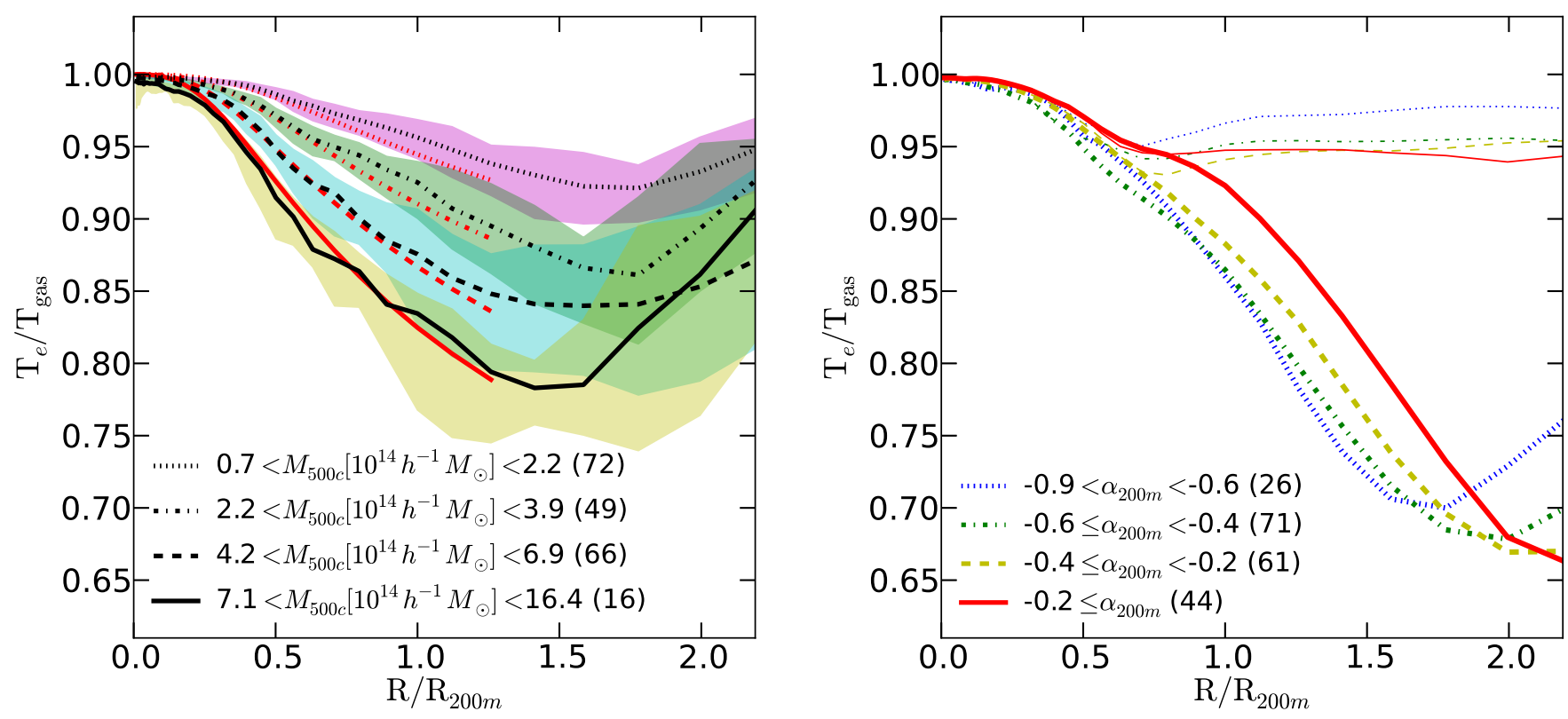

FIG. 1. - (Left) Black lines show the average profiles of the mass-weighted temperature bias binned by mass. We have selected 65 clusters at $z=0$ and their mainline progenitor data at $z=0.5,1$, and 1.5 prior to binning the profiles by mass. The number of clusters in each mass bin denoted in parentheses in the legend label. Red lines show the fitting function (Eqn. 7) with input masses that correspond to the average mass in each bin. (Right) Thick lines are the averaged profiles of the bias in the diffuse ICM, in bins of mass accretion rate (MAR) proxy $\alpha_{200 m}$; thin lines: the bias in the filament component in bins of $\alpha_{200 m}$. Filaments exhibit less of a temperature bias due to shorter equilibration times and decreased susceptibility to the accretion shock. Variations in the amount of recent accretion lead to scatter in the self-similar behavior of the temperature bias.

in Vikhlinin et al. (1998). We also exclude regions that correspond to overdense filamentary structures, the details of which are described in Avestruz et al. (2014).

\section{RESULTS}

\subsection{Dependence on mass and mass accretion rate}

The temperature bias from non-equilibrium electrons depends on the location of the accretion shock that generates the initial temperature difference between electrons and heavier ions and the relative age of the gas $(\sim M / \dot{M})$ compared with the equilibration timescale in Equation 2 The former depends on the mass accretion rate (MAR) of the cluster, and the latter depends on the the temperature and density (hence the mass) of the cluster.

The left panel of Figure 1 shows the profiles of the electron temperature bias $T_{e} / T_{\text {gas }}$ of the simulated clusters at $z=0,0.5,1,1.5$ averaged in four mass bins, with masses defined within $R_{200 m}$ (black lines). Note that we have taken the profiles of the 65 clusters at $z=0,0.5,1$, and 1.5 prior to binning the profiles by mass.

In a companion paper (Lau et al. 2014), we show that the average location of the accretion shock occurs at a fixed fraction of $R_{200 m}{ }^{2}$ independent of redshift. The location of the maximum temperature bias is mainly driven by the accretion shock, and therefore also exhibits the same redshift independence when scaled to $R_{200 m}$. The $T_{e} / T_{\text {gas }}$ profile for each mass bin shows a consistent location for the maximum temperature bias, which is located at the accretion shock radius $R_{\mathrm{sh}} \approx 1.6 \times R_{200 m}$. At this radius, the shocked electrons are maximally out of equilibrium with the heavier ions. The ex-

\footnotetext{
${ }^{2} R_{200 m}$ is the cluster radius enclosing an average density 200 times the mean matter density of the universe.
}

tent of the bias is larger for more massive clusters with hotter gas and longer equilibration times (see Equation 2).

The electron temperature bias also depends on the MAR of the cluster. The MAR shifts the location of the accretion shock that generates the non-equilibrium electrons. We use a proxy of the MAR of the cluster

$$
\alpha_{200 m} \equiv V_{r}^{D M}\left(r=R_{\alpha}\right) / V_{\text {circ }, 200 m},
$$

defined as the average mass-weighted dark matter radial velocity $V_{r}^{D M}$ measured at radius $R_{\alpha}=1.25 R_{200 \mathrm{~m}}$ and normalized by the circular velocity $V_{\text {circ }, 200 m} \equiv \sqrt{G M_{200 m} / R_{200 m}}$ of the cluster (Lau et al. 2014). A more negative $\alpha_{200 m}$ indicates higher mass accretion rate.

The value of $R_{\alpha}$ was chosen to minimize scatter relative to an alternative mass accretion rate proxy (Diemer \& Kravtsov 2014),

$$
\Gamma_{200 m}=\frac{\log _{10}\left(M_{200 m}(z=0) / M_{200 m}(z=0.5)\right)}{\log _{10}(a(z=0) / a(z=0.5))}
$$

which only describes the mass accretion rate of a cluster at $z=0$ (see Figure 5 in Lau et al. 2014). The choice of a fixed fraction of $R_{200 m}$ to measure an accretion rate provides a consistent way to compare accretion rates of clusters at different epochs.

The right-hand panel of Figure 1 shows the profile of the temperature bias in bins of $\alpha_{200 m}$. Following Lau et al. (2014), we decompose gas in a given radial bin into "filament" and "diffuse" components according to the density and radial velocity of the gas defined with respect to the cluster center. At large cluster-centric radii, a large fraction of the gas mass belongs to dense filaments and clumps, with net radial velocities that point towards the cluster center. The thick lines in the plot 
correspond to the bias in the diffuse ICM of the clusters, and the thin lines correspond to the bias in the filaments.

In the filament component of the gas (thin lines), the electron temperature is very close to the mean gas temperature. Gas in filaments also has higher densities and lower temperatures than gas in the diffuse component, leading to shorter equilibration times. In the diffuse component of the gas (thick lines), the location of maximum bias occurs closer to the cluster center for more rapidly accreting clusters. Clusters with higher accretion rates have more negative values of $\alpha_{200 m}$. Accreted material in these clusters have higher momentum fluxes, causing the location of accretion shock to move inward.

The distinction between the diffuse ICM and the filaments is visible in Figure 2 where we show slices of $T_{e} / T_{\mathrm{gas}}$ for a relaxed low mass cluster (left panel) and a merging massive cluster (right panel) from our simulation sample. The dashed circles indicate $R_{200 m}$ for each cluster. Darker shades in the maps indicate regions where more electrons are out of equilibrium with the heavier ions, corresponding to a larger temperature bias. The regions of larger bias mostly correspond to gas in the diffuse ICM. The lighter shades indicate regions where the electron temperature is very close to the mean gas temperature. Near $R_{200 m}$, the lighter shades correspond to gas in filaments, which experience shock heating at smaller radii than gas in the diffuse ICM.

In the more massive and less relaxed cluster shown in the right panel, mergers drive more small scale shocks that lead to more non-equilibrium electrons. Additionally, the higher ICM temperature in the outskirts of the more massive cluster leads to longer equilibration times and therefore larger temperature biases.

The temperature bias in the left panel of Figure 1 uses the mass-weighted temperatures from all of the cluster gas. Since filaments are denser in cluster outskirts, the temperature bias is more heavily weighted towards the filament contribution, where the non-equilibrium effect is small.

\subsection{Redshift dependence}

Figure 3 shows $T_{e} / T_{\text {gas }}$ as a function of cluster mass, both measured at $r=R_{200 m}$. Different redshifts $(z=0,0.5,1.0,1.5)$ are indicated by marker styles (respectively circles, squares, diamonds, and triangles). There is an apparent redshift dependence of $T_{e} / T_{\mathrm{gas}}$, with high- $z$ clusters exhibiting less of a bias than low $z$ clusters. However, the redshift dependence is primarily due to cluster mass growth. At low redshift, there are more high mass clusters with a more pronounced bias. For a given cluster mass, there is no significant trend of $T_{e} / T_{\text {gas }}$ with redshift. There is, however, a systematic trend in $T_{e} / T_{\text {gas }}$ with MAR. Clusters with higher MAR (more negative values of $\alpha_{200 m}$, as indicated by the color of the points in Figure 3 tend to a larger bias, with lower $T_{e} / T_{\text {gas }}$ values at $R_{200 m}$. As discussed in Section 3.1. clusters with higher MAR have accretion shocks closer to the cluster center, leading to an inward shift of the location of maximum bias.

We note that there can be additional redshift dependence in $T_{e} / T_{\mathrm{gas}}$ due to any evolution in the distribution of cluster assembly history. At a given redshift, more recently assembled clusters have more recently accreted electrons with less time to equilibrate; we expect the fraction of recently assembled clusters to vary with redshift. The mass accretion timescale $t_{\text {acc }} \equiv M / \dot{M}$ for a given cluster at a given redshift is an indicator of cluster age. Our MAR proxy, $\alpha_{200 m}$ (defined in Equation 5), is inversely proportional to $t_{\mathrm{acc}}$, as $V_{\mathrm{circ}, 200 \mathrm{~m}}$ is a proxy for cluster mass and $V_{r}^{D M}\left(r=R_{\alpha}\right)$ describes the instantaneous MAR. However, in our sample, there does not appear to be any strong correlation between $z$ and $\alpha_{200 m}$ for a given mass. To make a more stringent statement on any additional redshift dependence, we would need a larger box that samples a wider mass range in a given redshift bin.

\subsection{Fitting function of the temperature bias}

We provide a simple fitting function for the profile of the temperature bias:

$$
b_{e}(R) \equiv \frac{T_{e}(R)}{T_{\mathrm{gas}}(R)}=\frac{\left(x / x_{t}\right)^{-a}}{\left(1+\left(x / x_{t}\right)^{-b}\right)^{a / b}},
$$

where $x=R / R_{200 m}$ and the three best-fit parameters that account for the dependence on cluster mass are given by,

$$
\begin{aligned}
& x_{t}=0.629 \times\left(M_{200 m} / 10^{14} h^{-1} M_{\odot}\right)^{-0.1798} \\
& a=0.086 \times\left(M_{200 m} / 10^{14} h^{-1} M_{\odot}\right)^{0.3448} \\
& b=2.851 \times\left(M_{200 m} / 10^{14} h^{-1} M_{\odot}\right)^{-0.006} .
\end{aligned}
$$

In the left panel of Figure 1, we overplot profiles of the fitting function from Equation 7 in red. Each red line corresponds to the fitting function with an input mass that is the average cluster mass in each cluster mass bin. The fitting function adequately describe the averaged profiles of the bias, shown as black lines.

Here we do not decompose the ICM into diffuse and filament components, nor do we separately model dependence on $\alpha_{200 m}$, since applications of the fitting function likely treat spherical averages of the halo profile and do not typically have the information necessary to calculate $\alpha_{200 m}$. The dependence on $\alpha_{200 m}$ comes from the fact that accretion rate shifts the location of the shock radius. We have characterized the relationship between the shock radius and accretion rate in Figure 10 of the companion paper by Lau et al. (2014), which shows that the scatter in the shock radius at a given is $\alpha_{200 m}$ nearly a factor of two. This large scatter in the shock radius stems from highly aspherical and complex geometry at large radii. As a result, the location of the shock radius exhibits a relatively weak dependence (although systematic) on $\alpha_{200 m}$, whereas the mass dependence of the temperature bias exhibits a stronger behavior with better predictive power. We therefore use the fitting function to describe the bias in a statistical manner, parameterizing by mass alone.

\subsection{ICM profiles from mock $X$-ray maps}

$\mathrm{X}$-ray measurements of the ICM are only sensitive to the electron component of the plasma. A temperature bias due to non-equilibrium electrons propagates to ICM quantities inferred from the X-ray temperature.

We measure the X-ray temperature profiles from two sets of mock Chandra maps: one generated with the mean gas temperature, and the other with the electron temperature. This allows us to compare the temperature difference accounting for instrumental response, projection effects, and the spectroscopic weighting of the X-ray gas.

Figure 4 shows the bias in the X-ray projected temperature profile, measured from spectral fitting of photons from each of the two mock maps. The bias is then calculated as a ratio of measured X-ray electron temperature to the X-ray temperature that would be measured if electrons were in equilibrium with heavier ions. Red data points show the projected 

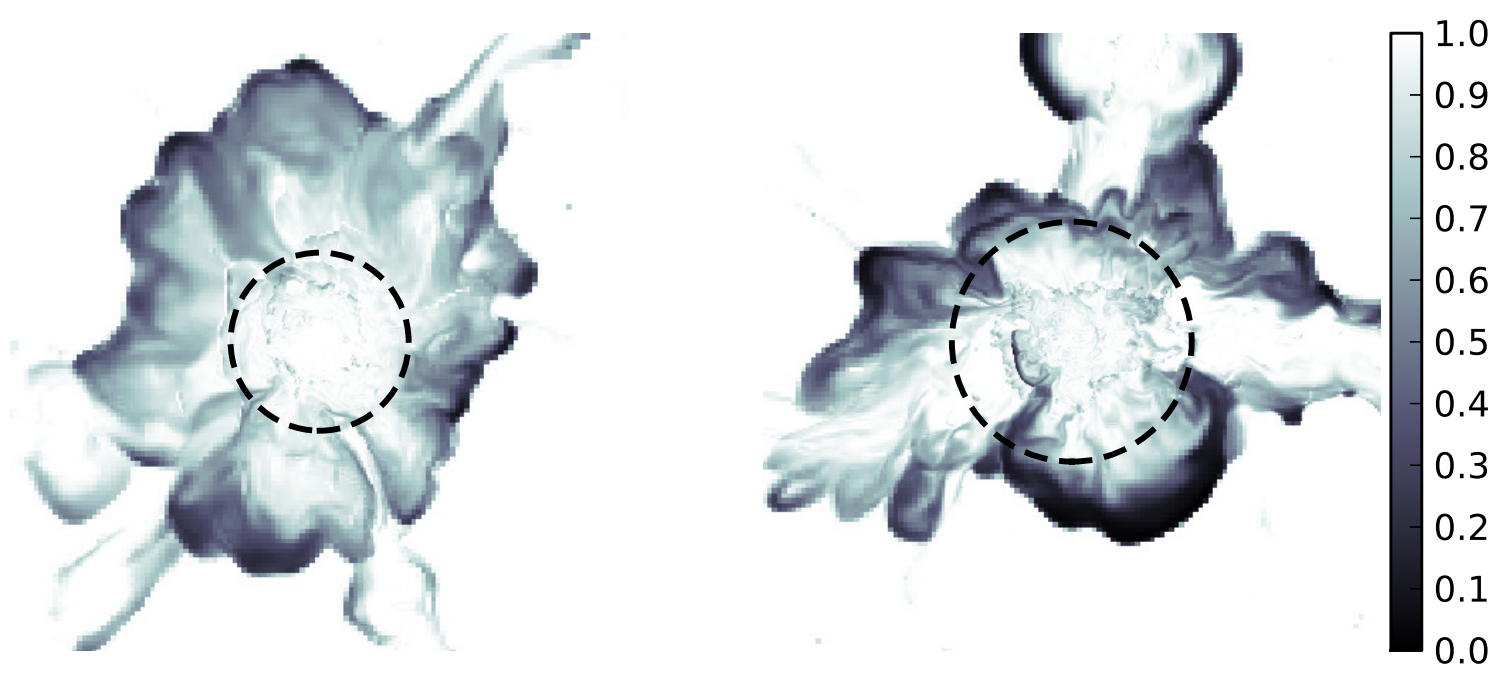

FIG. 2.- Sliced maps of $T_{e} / T_{\text {gas }}$ for the most relaxed and least massive cluster (left panel), and the most massive and least relaxed cluster (right panel) from our simulation. The colorbar indicates $T_{e} / T_{\text {gas }}$. The dimension for each panel is $15.6 h^{-1} \mathrm{Mpc} \times 15.6 h^{-1} \mathrm{Mpc}$, with depth of $7.6 h^{-1} \mathrm{kpc}$. The circle in dashed line shows $R_{200 m}$ of the cluster. Between $1.0<R / R_{200 m}<1.5, T_{e} / T_{\text {gas }}$ is close to unity in the filamentary gas with high momentum flux entering the cluster well, but is significantly less than unity in the diffuse gas at the same cluster-centric radii. The right panel shows gas from the bottom-left filament experiencing an accretion shock at radii smaller than $R_{200 m}$.

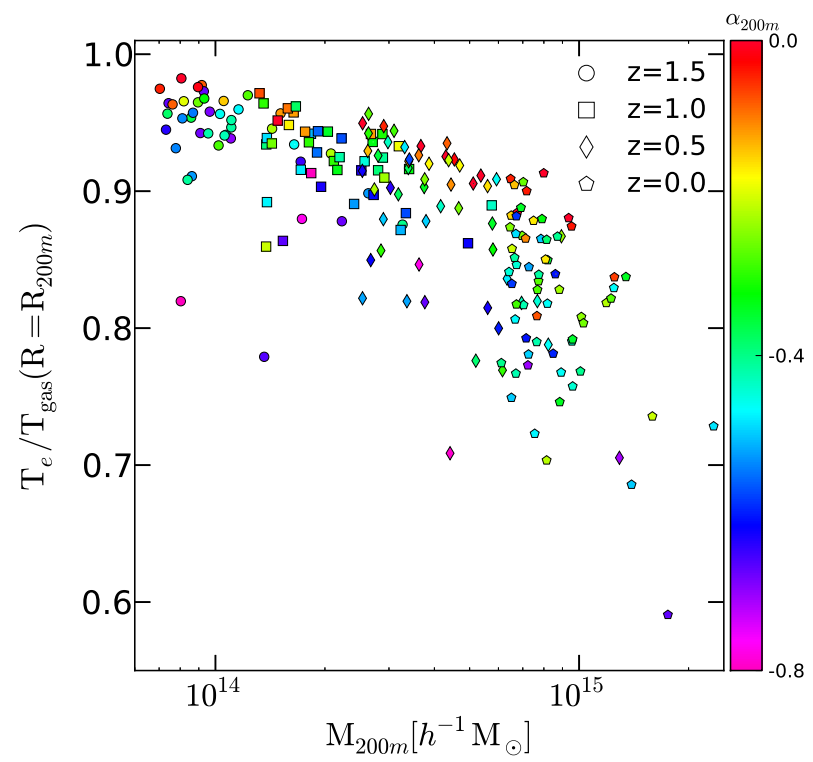

FIG. 3.- The temperature bias of non-equilibrium electrons as a function of cluster mass, both measured at $r=R_{200 m}$. We show the bias for all 65 clusters at $z=0,0.5,1$, and 1.5 , with respective marker styles of circles, squares, diamonds, and triangles. For each data point, we color code the value of the mass accretion rate parameter, $\alpha_{200 m}$.

X-ray temperature bias for one of the less massive relaxed clusters with $T_{X}=5.96 \mathrm{keV}\left(M_{200 m}=7.2 \times 10^{14} h^{-1} M_{\odot}\right)$ and $\alpha_{200 m}=-0.05$. Blue data points show the same bias in one of our more massive unrelaxed clusters with $T_{X}=11.03 \mathrm{keV}$ $\left(M_{200 m}=1.75 \times 10^{15} h^{-1} M_{\odot}\right)$ and $\alpha_{200 m}=-0.66$. These two representative clusters bracket the effects of non-equilibrium

electrons on X-ray measurements in this mass range, but represent upper limits of the temperature bias in both cases. For the relaxed less massive cluster, the bias within $R<R_{500 c}$ is less than $5 \%$. In the unrelaxed, more massive cluster that has experienced rapid recent accretion, the bias is $\sim 10 \%$ at $R_{500 c}$, and increases precipitously at larger radii.

We have also overplotted the ratio of the mass-weighted electron temperature profile to the mass-weighted mean temperature profile of the diffuse ICM, computed directly from the simulation. The agreement between the bias in mock Xray measurements and the bias in the mass-weighted temperature shows that the bias is comparable between the two methods of measuring the ICM temperature.

Note that the upturn in the bias at $R \approx 0.8 R_{200 m}$ for the red mock X-ray data in the less massive cluster is due to line of sight effects; the bias is not perfectly spherical, even in the bulk component. Some projections will have a bias in the projected X-ray temperature that is either below or above the bias calculated from the spherically averaged mass-weighted temperatures shown in the red dotted line. In this projection, there is slightly less shock heated material at this radius compared to other lines of sight.

Likewise, the drop in the X-ray temperature bias for the more massive less relaxed cluster at $R \approx 0.8 R_{200 m}$ is also due to the line of sight; the line of sight for the X-ray data has more shock heated material at that radius compared to other lines of sight, all of which have a mass-weighted average shown in the solid blue line.

To illustrate the scatter in projected temperature biases, we have also plotted, in lighter colors, the measured X-ray temperature bias along two other orthogonal projections for each cluster. In both clusters, the temperature biases are generally present in all three projections. However, the temper- 


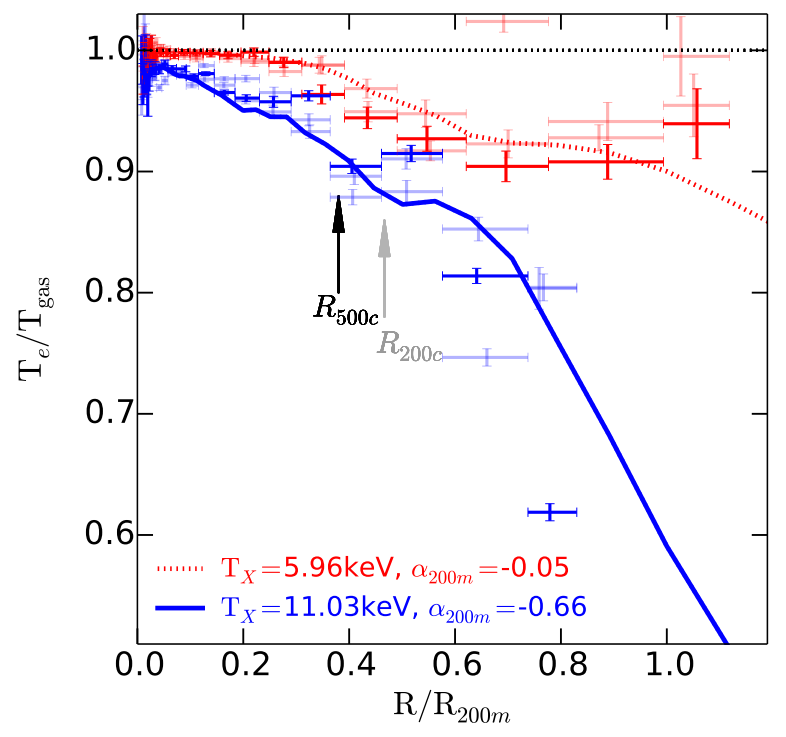

FIG. 4.- Lines show the temperature bias profiles of the diffuse ICM in one of our most massive and least relaxed clusters (blue solid), and one of our least massive and most relaxed clusters (red dotted). Data points show the corresponding bias of the diffuse ICM measured from mock Chandra Xray maps. The light red and blue data points correspond to the respective temperature biases of the least massive and most relaxed cluster along two other orthogonal projections to illustrate line-of-sight variations. The black and grey arrows denote $R_{500 c}$ and $R_{200 c}$ respectively.

TABLE 1

BIASES IN HYDROSTATIC MASS AND GAS TEMPERATURE OF GALAXY CLUSTERS AT $z=0$

\begin{tabular}{cccc}
\hline$T_{X}[\mathrm{keV}]$ & $R=R_{500 c}$ & $R=R_{200 c}$ & $R=R_{200 m}$ \\
\hline \multicolumn{5}{c}{ (a) $M_{\mathrm{HSE}}\left(R ; T_{e}\right) / M_{\mathrm{HSE}}\left(R ; T_{\mathrm{gas}}\right)$} \\
\hline $4.2-6.1(38)$ & $0.993 \pm 0.002$ & $0.959 \pm 0.007$ & $0.889 \pm 0.009$ \\
$6.1-8.7(24)$ & $0.989 \pm 0.005$ & $0.926 \pm 0.010$ & $0.868 \pm 0.012$ \\
$8.7-12.5(3)$ & $0.961 \pm 0.009$ & $0.893 \pm 0.016$ & $0.803 \pm 0.041$ \\
\hline \multicolumn{5}{c}{ (b) core excised $b_{e}(<R) \equiv T_{e}(<R) / T_{\mathrm{gas}}(<R)$} \\
\hline $4.2-6.1(38)$ & $0.991 \pm 0.001$ & $0.985 \pm 0.002$ & $0.975 \pm 0.001$ \\
$6.1-8.7(24)$ & $0.985 \pm 0.002$ & $0.976 \pm 0.003$ & $0.967 \pm 0.003$ \\
$8.7-12.5(3)$ & $0.972 \pm 0.003$ & $0.962 \pm 0.003$ & $0.952 \pm 0.004$ \\
\hline
\end{tabular}

Note. - Panel (a) shows the mean biases in hydrostatic mass and their $1 \sigma$ errors due to non-equilibrium electrons at the given radius $R$. Panel (b) shows the mean bias the mass-weighted temperature integrated between $0.15 R_{500 \mathrm{c}}$ and $R$ specified in the first row. The number in the parentheses indicate the number of clusters in each bin of $T_{X}$.

ature measurements from X-ray mocks occasionally include photons from clumps and filaments that were not completely masked out; these photons can contribute to the projected annular average by driving the temperature ratio closer to unity, since the high-density gas in clumps and filaments has less of a temperature bias.

\subsection{The effects on hydrostatic mass estimates and scaling relations}

We calculate the corresponding effect of non-equilibrium electrons on hydrostatic mass estimates for our cluster sam- ple in four temperature bins. In Table 1, we list the ratios between the hydrostatic mass estimates calculated with electron temperature to that with the mean gas temperature. The hydrostatic mass estimate from the electron temperature corresponds to the observationally inferred mass in the presence of non-equilibrium electrons, while the hydrostatic mass estimate from the mean gas temperature corresponds to the inferred mass with electrons fully in equilibrium with the heavier ions.

In the most massive clusters $\left(T_{X} \gtrsim 8 \mathrm{keV}\right)$, the core-excised mass-weighted electron temperature is biased low by $3 \%$ at $R_{500 c}$, and as much as 5\% at $R_{200 m}$. The corresponding bias in the observed hydrostatic mass for these clusters is $4 \%$ at $R_{500 c}$, and $20 \%$ at $R_{200 m}$. The bias in observed hydrostatic mass is larger than the bias in the temperature, $b_{e}(R)$, at each of these radii, as the hydrostatic mass within a radius is proportional to both the temperature and the temperature gradient at that radius.

Table 1 also lists the resulting biases in mass-weighted cluster temperature, $b_{e}(<R) \equiv T_{e}(<R) / T_{\text {gas }}(<R)$, measured out to three different values of $R: R_{500 c}, R_{200 c}$, and $R_{200 m} . b_{e}(<R)$ is an core-excised integrated quantity between the $0.15 R_{500 c}$ and the maximum given radius, $R . b_{e}(<R)$ have a smaller bias than the temperature bias $b_{e}(R)$ measured at $R$ since the temperature bias monotonically increases until the shock radius. The bias in the core-excised temperature is $<3 \%$ at $R_{500 c}$ and $<5 \%$ at $R_{200 m}$, smaller than the biases in both the electron temperature at those radii and the observed hydrostatic mass.

Since the temperature bias due to non-equilibrium electrons has a larger effect on the hydrostatic mass estimate than on the integrated temperature, scaling relations such as the $M-T_{X}$ and $M-Y_{X}$ will have a shallower slope with increasing cluster mass, leading to deviations from self-similarity.

\section{SUMMARY AND DISCUSSION}

We have used a mass-limited sample of 65 galaxy clusters simulated in a cosmological volume to characterize the effects of non-equilibrium electrons on gas temperature in the outskirts of galaxy clusters. In this work, we quantified the dependence of the temperature bias $T_{e} / T_{\mathrm{gas}}$ on cluster mass and dynamical state in both the diffuse and filamentary components of the ICM in cluster outskirts. We provided a mass-dependent fitting function for $T_{e} / T_{\mathrm{gas}}$, which will be useful in quantifying the astrophysical uncertainties due to nonequilibrium electrons, and in placing constraints on physical processes in accretion shocks. Additionally, we used mock Chandra X-ray maps with and without non-equilibrium electrons to determine how the temperature bias manifests in ICM profiles derived from X-ray observations.

To summarize our findings:

- The temperature bias from non-equilibrium electrons in the diffuse component of the ICM can reach $40 \%$ within $R \leq R_{200 m}$, and is dependent on the mass and mass accretion rate of the cluster. More massive clusters have longer equilibration times due to their higher gas temperatures, leading to a more strongly biased electron temperature at all radii. Clusters with higher mass accretion rates have smaller accretion shock radius, leading to stronger bias at any given radius inside the shock.

- The bias is azimuthally asymmetric. Due to lower temperatures, higher densities, and a net momentum flux towards the cluster center, the effects of non- 
equilibrium electrons in gas filaments is much smaller than in the diffuse component of the ICM.

- Non-equilibrium electrons can affect X-ray measurements of galaxy cluster outskirts by biasing the projected X-ray temperatures low. The magnitude of the temperature bias accounting for instrumental response, projection effects, and spectroscopic weighting is similar to the temperature bias measured directly from simulations.

- Non-equilibrium electrons introduce biases in the hydrostatic mass estimates as well as the average cluster temperature of massive clusters. In the hottest $\left(T_{X} \gtrsim\right.$ $8 \mathrm{kev}$ ) clusters, the hydrostatic mass is biased low by $4 \%$ at $R_{500 c}$ and $20 \%$ at $R_{200 m}$, while the core-exicsed mass-weighted temperature is biased low by $3 \%$ and $5 \%$, respectively. Since the bias in the hydrostatic mass estimates is larger than the bias in the average cluster temperatures and the biases are larger for hotter clusters, the presence of non-equilibrium electrons leads to shallower slopes in the high mass end of the $M-T_{X}$ and $M-Y_{X}$ scaling relations than self-similar predictions.

- We provide a mass dependent fitting function for the profile of the temperature bias (Equation77). The fitting function can be useful in the theoretical modeling of electron-ion equilibration in galaxy clusters.

Our results have implications for X-ray and SZ observations of cluster outskirts. The temperature bias due to nonequilibrium electrons leads to underestimates in entropy, pressure, and hydrostatic mass that are of the same size as the temperature bias. Since gas entropy is directly proportional to gas temperature, the temperature bias of order $\lesssim 15 \%$ at $r \gtrsim R_{200 c}$ can partially explain the flattening of entropy observed by Suzaku, in addition to gas clumping that biases the density high and entropy low. The effects of nonequilibrium electrons must also be considered when interpreting the recent Planck stacked SZ measurements of electron pressure profiles, which measured the pressure profiles out to $3 \times R_{500 c} \approx 1.2 R_{200 m}$ (Planck Collaboration Int. V [2013). The effect of non-equilibrium electrons is mass dependent; the corresponding temperature bias could lead to deviations from self-similarity in mass-observable scaling relations.

The fitting function provided in Equation 7 can also be useful in bracketing the effects of non-equilibrium electrons on measurements of the SZ power spectrum (Hill \& Pajer 2013) and SZ bispectrum (Hill \& Sherwin 2013; Crawford et al. 2014). The temperature bias will be especially significant in the SZ bispectrum, since the bispectrum is most sensitive to massive clusters at low redshift (Bhattacharya et al. 2012), whose outskirts would have the largest temperature bias due to non-equilibrium electrons.

Our quantitative study of non-equilibrium electrons in galaxy cluster outskirts assumes a maximal equilibration Spitzer timescale. Our model assumes negligible electron heating within shocks, but there can be non-adiabatic heating due to plasma instabilities (e.g., Bykov et al.|2008). However, these are expected to be small in the accretion shocks whose Mach numbers are high. The results presented in this work thus provide an upper limit of the temperature differences between ions and electrons.

While the temperature bias in high Mach number accretion shocks is not directly measurable from current observational data, there has been some observational evidence indicating a rapid equilibration timescale in low Mach number merger shocks by measuring the width of the merger shock in colliding galaxy clusters (e.g., Markevitch 2006). The equilibration timescale can be constrained by measuring the temperature jump across the shock. An equilibration timescale that is significantly shorter than the Coulomb timescale will lead to a sharper electron temperature jump, more similar to the temperature jump of the heavier ions. A similar observational test is possible for accretion shocks in cluster outskirts. However, accretion shocks are too faint to detect with the current generation of X-ray telescopes, and such measurements will not be possible until the next generation of X-ray missions.

Since non-equilibrium electrons have a progressively larger effect on ICM temperature measurements at increasing cluster-centric radii, non-equilibrium physics can have an impact on the electron pressure profiles in clusters outskirts that have recently become accessible to SZ observations, e.g, with Planck. Detailed comparisons of observed and simulated pressure profiles can therefore provide further observational constraints on the equilibration efficiency between electrons and protons.

Finally, one can also assess the non-equilibrium state of other ions by measuring line intensity ratios of different metals with high-resolution X-ray spectroscopy onboard ASTRO$H^{3}$ Future X-ray missions, such as Athena $++^{4}$ and SMART-X will further extend the study of the non-equilibrium phenomena into the virialization regions of the outskirts of galaxy clusters and the intergalactic medium.

We thank Xun Shi, Eiichiro Komatsu, and the anonymous referee for useful discussion and comments on the manuscript. This work is supported by NSF grant AST1412768 \& 1009811, NASA ATP grant NNX11AE07G, NASA Chandra grants GO213004B and TM4-15007X, and by the facilities and staff of the Yale University Faculty of Arts and Sciences High Performance Computing Center. CA acknowledges support from the NSF Graduate Student Research Fellowship and Alan D. Bromley Fellowship from Yale University.

\section{REFERENCES}

Allen, S. W., Evrard, A. E., \& Mantz, A. B. 2011, ARA\&A $49 \mid 409$ Avestruz, C., Lau, E. T., Nagai, D., \& Vikhlinin, A. 2014, ApJ 791\|117 Bautz, M. W., Miller, E. D., Sanders, J. S., et al. 2009, PASJ, 61 1117 Bhattacharya, S., Nagai, D., Shaw, L., Crawford, T., \& Holder, G. P. 2012,

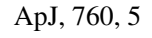

\footnotetext{
${ }^{3}$ http://astro-h.isas.jaxa.jp/en/

4 http://athena2.irap.omp.eu/

5 http://smart-X.cfa.harvard.edu/
}

Bykov, A. M., Paerels, F. B. S., \& Petrosian, V. 2008, Space Sci. Rev.|134. 141

Crawford, T. M., Schaffer, K. K., Bhattacharya, S., et al. 2014, ApJ $784 \mid 143$ Diemer, B., \& Kravtsov, A. V. 2014, ApJ $789|1|$

Ettori, S., \& Fabian, A. C. 1998, MNRAS 293 L33

Fox, D. C., \& Loeb, A. 1997, ApJ, 491|459

Hill, J. C., \& Pajer, E. 2013, Phys. Rev. D 88063526

Hill, J. C., \& Sherwin, B. D. 2013, Phys. Rev. D 87023527

Hoshino, A., Henry, J. P., Sato, K., et al. 2010, PASJ, $62 \mid 371$ 
Kaastra, J. S., \& Jansen, F. A. 1993, A\&AS, 97873

Kawaharada, M., Okabe, N., Umetsu, K., et al. 2010, ApJ 714.423

Kravtsov, A. V. 1999, PhD thesis, New Mexico State Univ.

Kravtsov, A. V., Klypin, A., \& Hoffman, Y. 2002, ApJ 571. 563

Lau, E. T., Nagai, D., Avestruz, C., Nelson, K., \& Vikhlinin, A. 2014, ArXiv e-prints, arXiv 1411.5361

Liedahl, D. A., Osterheld, A. L., \& Goldstein, W. H. 1995, ApJ 438, L115

Mantz, A., Allen, S. W., Rapetti, D., \& Ebeling, H. 2010, MNRAS 406. 1759

Markevitch, M. 2006, in ESA Special Publication, Vol. 604, The X-ray Universe 2005, ed. A. Wilson, 723, astro-ph/0511345

McDonald, M., Benson, B. A., Vikhlinin, A., et al. 2014, ApJ 79467

Mewe, R., Gronenschild, E. H. B. M., \& van den Oord, G. H. J. 1985, A\&AS, 62.197

Nagai, D., \& Lau, E. T. 2011, ApJ 731 L10

Nagai, D., Vikhlinin, A., \& Kravtsov, A. V. 2007, ApJ $655 \mid 98$

Nelson, K., Lau, E. T., \& Nagai, D. 2014a, ApJ 792. 25

Nelson, K., Lau, E. T., Nagai, D., Rudd, D. H., \& Yu, L. 2014 b, ApJ 782. 107

Planck Collaboration, Ade, P. A. R., Aghanim, N., et al. 2014, A\&A 571 A20

Planck Collaboration Int. V. 2013, A\&A 550 A131
Reiprich, T. H., Hudson, D. S., Zhang, Y.-Y., et al. 2009, A\&A. 501. 899 Roncarelli, M., Ettori, S., Borgani, S., et al. 2013, MNRAS 4323030 Rudd, D. H., \& Nagai, D. 2009, ApJ 701. L16

Rudd, D. H., Zentner, A. R., \& Kravtsov, A. V. 2008, ApJ 672 19

Shi, X., \& Komatsu, E. 2014, MNRAS 442.521

Shi, X., Komatsu, E., Nelson, K., \& Nagai, D. 2014, ArXiv e-prints, arXiv 1408.3832

Simionescu, A., Allen, S. W., Mantz, A., et al. 2011, Science 331, 1576

Spitzer, L. 1962, Physics of Fully Ionized Gases (New York: Interscience)

Tozzi, P., \& Norman, C. 2001, ApJ 546 63

Urban, O., Simionescu, A., Werner, N., et al. 2014, MNRAS 437.3939

Vazza, F., Eckert, D., Simionescu, A., Brüggen, M., \& Ettori, S. 2013, MNRAS 429.799

Vikhlinin, A., McNamara, B. R., Forman, W., et al. 1998, ApJ 502.558

Vikhlinin, A., Kravtsov, A. V., Burenin, R. A., et al. 2009, ApJ $692 \mid 1060$

Voit, G. M., Balogh, M. L., Bower, R. G., Lacey, C. G., \& Bryan, G. L. 2003, ApJ 593272

Walker, S. A., Fabian, A. C., Sanders, J. S., Simionescu, A., \& Tawara, Y. 2013, MNRAS 432554

Wong, K.-W., \& Sarazin, C. L. 2009, ApJ 707. 1141

Zhuravleva, I., Churazov, E., Kravtsov, A., et al. 2013, MNRAS 428 |3274 